\title{
RESOLUTION OF TEMPERATURE PROBLEMS BY THE USE OF FINITE FOURIER TRANSFORMATIONS
}

\author{
HERBERT KAPFEL BROWN ${ }^{1}$
}

1. The finite sine transformation of the convolution. The finite sine transformation and the finite cosine transformation of $U(x)$ with respect to $x$ are defined ${ }^{2}$ by

and

$$
u_{s}(n)=\int_{0}^{\pi} U(x) \sin n x d x=S\{U(x)\}
$$

$$
u_{c}(n)=\int_{0}^{\pi} U(x) \cos n x d x=C\{U(x)\},
$$

respectively. ${ }^{3}$ In particular, $S\left\{U^{\prime}\right\}=-n C\{U\}$, and $S\left\{U^{\prime \prime}\right\}$ $=-n\left[(-1)^{n} U(\pi)-U(0)\right]-n^{2} S\{U\}$. Further, if $U(x)$ and $U^{\prime}(x)$ vanish at the end points of the interval $(0, \pi)$, then $S\left\{U^{\prime \prime}\right\}=-n^{2} S\{U\}$, and $C\left\{U^{\prime}\right\}=n S\{U\}$.

The convolution $U_{1} * U_{2}$, or Faltung, ${ }^{2}$ of the two functions $U_{1}(x)$, $-2 \pi \leqq x \leqq 2 \pi$, and $U_{2}(x),-\pi \leqq x \leqq \pi$, is defined as follows:

$$
U_{1} * U_{2}=\int_{-\pi}^{\pi} U_{1}(x-\xi) U_{2}(\xi) d \xi .
$$

It is evident that the convolution of two even functions is an even function, that the convolution of two odd functions is an even function, and that the convolution of an odd function and an even function is an odd function.

The following theorem is proved by Kniess: 4

THEOREM. If $U_{1}(x),-2 \pi \leqq x \leqq 2 \pi$, and $U_{2}(x),-\pi \leqq x \leqq \pi$, are bounded and integrable, if $U_{1}$ is odd and periodic with period $2 \pi$, and if $U_{2}$ is even, then 1944.

Presented to the Society, September 10,1942; received by the editors March 8 ,

${ }^{1}$ The author wishes to thank the referee and Professor R. V. Churchill for many valuable suggestions.

${ }^{2} \mathrm{G}$. Doetsch, Integration von Differentialgleichungen vermittels der endlichen Fourier Transformation, Math. Ann. vol. 112 (1935) pp. 52-68.

${ }^{3}$ The lower case letters will be used to signify the transforms of the functions designated by the corresponding capital letters. Instead of using the symbols $u_{s}(n)$ and $u_{c}(n)$ we shall use $u(n)$ for both, whenever it is evident which one is meant.

${ }^{4}$ Hans Kniess, Lösung von Randwertproblemen bei Systemen gewöhnlicher Differentialgleichungen vermittels der endlichen Fourier Transformation, Math. Zeit. vol. 44 (1938) pp. 266-291. 


$$
S\left\{U_{1} / 2 * U_{2}\right\}=S\left\{U_{1}\right\} C\left\{U_{2}\right\} .
$$

2. Temperature distribution in a bar. Consider a cylindrical bar of length $\pi$ which has a diameter so small that the variation of temperature $U(x, t)$ over every cross section can be neglected. The thermal conductivity of the bar is a function of the time. There is a continuous internal source of heat along the bar and the initial temperature at each point of the bar is given by a prescribed function. The rate of loss of heat through the surface at each point is proportional to the temperature at that point. The temperatures at the ends $x=0$ and $x=\pi$ are determined by prescribed functions of $t$.

The boundary value problem for the temperature $U(x, t)$ can be written

$$
\begin{aligned}
L(U) & \equiv \partial U / \partial t-C_{1}(t) \partial^{2} U / \partial x^{2}+C_{2}(t) U=P(x, t), \\
& 0<x<\pi, t>0, \\
U(+0, t)=C_{3}(t), & U(\pi-0, t)=C_{4}(t), \\
U(x,+0)=F(x), & 0<x<\pi .
\end{aligned}
$$

The prescribed functions $P, F, C_{1}, C_{2}, C_{3}$, and $C_{4}$ are assumed to satisfy the following conditions: $P$ and its first three derivatives with respect to $x$ are continuous functions of $x$ and $t$ for all values of $x$ and $t$ in $0 \leqq x \leqq \pi, t \geqq 0 ; F$ and its first three derivatives with respect to $x$ are continuous in $x$ for $0 \leqq x \leqq \pi ; P, F$, and their first two derivatives with respect to $x$ vanish at the end points of the interval $(0, \pi) ; C_{1}$ and $C_{2}$ are positive and continuous for $t \geqq 0 ; C_{3}^{\prime}$ and $C_{4}^{\prime}$ are continuous for $t \geqq 0 ; C_{3}(0)=C_{4}(0)=0$.

3. Resolution of temperature problem. It is obvious that the solution of our boundary value problem can be written

$$
U(x, t)=U_{1}(x, t)+U_{2}(x, t)+U_{3}(x, t)+U_{4}(x, t),
$$

where $U_{1}, U_{2}, U_{3}$, and $U_{4}$ are solutions of the problems:

$$
\begin{array}{ll}
L\left(U_{1}\right)=P(x, t), & U_{1}(+0, t)=0, \\
U_{1}(\pi-0, t)=0, & U_{1}(x,+0)=0 ; \\
L\left(U_{2}\right)=0, & U_{2}(+0, t)=C_{3}(t), \\
U_{2}(\pi-0, t)=0, & U_{2}(x,+0)=0 ; \\
L\left(U_{3}\right)=0, & U_{3}(+0, t)=0, \\
U_{3}(\pi-0, t)=C_{4}(t), & U_{3}(x,+0)=0 ;
\end{array}
$$

and 


$$
\begin{array}{ll}
L\left(U_{4}\right)=0, & U_{4}(+0, t)=0, \\
U_{4}(\pi-0, t)=0, & U_{4}(x,+0)=F(x) .
\end{array}
$$

If in problem $\left(\mathrm{A}_{2}\right)$ we replace $C_{3}(t)$ by $C_{4}(t)$ and $x$ by $\pi-x$, then $U_{2}(x, t)$ reduces to $U_{3}(x, t)$. For this reason we shall not have to consider problem $\left(\mathrm{A}_{3}\right)$ as a problem to be solved.

The finite sine transformation with respect to $x$ applied to the first and fourth equations in problems $\left(A_{1}\right),\left(A_{2}\right)$, and $\left(A_{4}\right)$ transforms each of these boundary value problems into a problem in linear ordinary differential equations of the first order which can be written as follows:

$$
\begin{gathered}
d\left[u_{1}(n, t)\right] / d t+\left[n^{2} C_{1}(t)+C_{2}(t)\right] u_{1}(n, t)=p(n, t), \\
u_{1}(n,+0)=0 ; \\
d\left[u_{2}(n, t)\right] / d t+\left[n^{2} C_{1}(t)+C_{2}(t)-n C_{1}(t) C_{3}(t)\right] u_{2}(n, t)=0, \\
u_{2}(n,+0)=0 ;
\end{gathered}
$$

and

(A $\left.\mathrm{A}_{4}^{\prime}\right) \quad d\left[u_{4}(n, t)\right] / d t+\left[n^{2} C_{1}(t)+C_{2}(t)\right] u_{4}(n, t)=0, u_{4}(n,+0)=f(n)$.

The solutions $u_{1}(n, t), u_{2}(n, t)$, and $u_{4}(n, t)$ of the problems $\left(\mathrm{A}_{1}^{\prime}\right)$, $\left(\mathrm{A}_{2}^{\prime}\right)$, and $\left(\mathrm{A}_{4}^{\prime}\right)$, respectively, are given as follows:

$$
\begin{aligned}
& u_{1}(n, t)=\int_{0}^{t} p(n, \tau) \exp \left[-n^{2} \sigma_{1}(t, \tau)\right] \exp \left[-\sigma_{2}(t, \tau)\right] d \tau, \\
& u_{2}(n, t)=\int_{0}^{t} C_{3}(\tau) C_{1}(\tau) \exp \left[-n^{2} \sigma_{1}(t, \tau)\right] \exp \left[-\sigma_{2}(t, \tau)\right] d \tau,
\end{aligned}
$$

and

$$
u_{4}(n, t)=f(n) \exp \left[-n^{2} s_{1}(t)\right] \exp \left[-s_{2}(t)\right],
$$

where we have made the definitions: $s_{i}(t)=\int_{0}^{t} C_{i}(\xi) d \xi, \sigma_{i}(t, \tau)=s_{i}(t)$ $-s_{i}(\tau), 0 \leqq \tau \leqq t$.

It will now be shown that each of the problems $\left(A_{1}\right),\left(A_{2}\right)$, and $\left(A_{4}\right)$ can be further resolved into a single simple boundary value problem. That is to say, each of the solutions $u_{1}, u_{2}$, and $u_{4}$ can be expressed in terms of $u_{0}(n, t)$, the transform of $U_{0}(x, t)$, which is the solution of the following boundary value problem:

$$
\begin{array}{rlrl}
\partial U_{0} / \partial t & =\partial^{2} U_{0} / \partial x^{2}, & 0<x<\pi, t & >0, \\
U_{0}(+0, t) & =0, \quad U_{0}(\pi-0, t)=0, & t>0, \\
U_{0}(x,+0) & =(\pi-x) / \pi, & 0<x<\pi .
\end{array}
$$


Making a formal application of the finite sine transformation to (B) gives

$$
d\left[u_{0}(n, t)\right] / d t+n^{2} u_{0}(n, t)=0, \quad u_{0}(n,+0)=1 / n .
$$

The solution of this transformed problem is

$$
u_{0}(n, t)=(1 / n) \exp \left(-n^{2} t\right) .
$$

From this solution we obtain the following identities:

$$
\begin{aligned}
\exp \left[-n^{2} s_{1}(t)\right] & =n u_{0}\left[n, s_{1}(t)\right], \\
n \exp \left[-n^{2} \sigma_{1}(t, \tau)\right] C_{1}(\tau) & =\partial u_{0}\left[n, \sigma_{1}(t, \tau)\right] / \partial \tau, \\
n \exp \left[-n^{2} s_{1}(t)\right] C_{1}(t) & =-\partial u_{0}\left[n, s_{1}(t)\right] / \partial t .
\end{aligned}
$$

The transforms $u_{1}(n, t), u_{2}(n, t)$, and $u_{4}(n, t)$ can now be expressed as functions of $u_{0}(n, t)$ as follows:

$$
\begin{aligned}
& u_{1}(n, t)=\int_{0}^{t} \exp \left[-\sigma_{2}(t, \tau)\right] p(n, \tau) n u_{0}\left[n, \sigma_{1}(t, \tau)\right] d \tau, \\
& u_{2}(n, t)=\int_{0}^{t} C_{3}(\tau) \exp \left[-\sigma_{2}(t, \tau)\right] \frac{\partial}{\partial \tau} u_{0}\left[n, \sigma_{1}(t, \tau)\right] d \tau,
\end{aligned}
$$

and

$$
u_{4}(n, t)=\exp \left[-s_{2}(t)\right] f(n) n u_{0}\left[n, s_{1}(t)\right] .
$$

If the convolution theorem is now applied to $u_{1}(n, t)$ and $u_{4}(n, t)$, a formal inverse sine transformation of $u_{1}(n, t), u_{2}(n, t)$, and $u_{4}(n, t)$ can be performed. The result can be written

$$
\begin{aligned}
U_{1}(x, t)= & \frac{1}{2} \int_{0}^{t} \exp \left[-\sigma_{2}(t, \tau)\right] \\
& \cdot \int_{-\pi}^{\pi} P(x-\xi, \tau) \frac{\partial}{\partial \xi} U_{0}\left[\xi, \sigma_{1}(t, \tau)\right] d \xi d \tau, \\
U_{2}(x, t)= & \int_{0}^{t} C_{3}(\tau) \exp \left[-\sigma_{2}(t, \tau)\right] \frac{\partial}{\partial \tau} U_{0}\left[x, \sigma_{1}(t, \tau)\right] d \tau,
\end{aligned}
$$

and

$$
U_{1}(x, t)=\frac{1}{2} \exp \left[-s_{2}(t)\right] \int_{-\pi}^{\pi} F(x-\xi) \frac{\partial}{\partial \xi} U_{0}\left[\xi, s_{1}(t)\right] d \xi,
$$

where $P$ and $F$ are the odd, periodic extensions of period $2 \pi$ of the original functions $P$ and $F$, respectively, and $\partial U_{0} / \partial \xi$ is the even ex- 
tension of the original function $\partial U_{0} / \partial \xi$. The solution $U_{3}$ of problem $\left(A_{3}\right)$ can be written

$$
U_{3}(x, t)=\int_{0}^{t} C_{4}(\tau) \exp \left[-\sigma_{2}(t, \tau)\right] \frac{\partial}{\partial \tau} U_{0}\left[\pi-x, \sigma_{1}(t, \tau)\right] d \tau .
$$

THEOREM. The general temperature problem (A) can be resolved into the solution of the simple temperature problem (B) by formulas (1) and (2). That is,

$$
U(x, t)=\frac{1}{2} \int_{0}^{t} \exp \left[-\sigma_{2}(t, \tau)\right] \int_{-\pi}^{\pi} P(x-\xi, \tau) \frac{\partial}{\partial \xi} U_{0}\left[\xi, \sigma_{1}(t, \tau)\right] d \xi d \tau
$$

$$
\begin{aligned}
& +\int_{0}^{t} C_{4}(\tau) \exp \left[-\sigma_{2}(t, \tau)\right] \frac{\partial}{\partial \tau} U_{0}\left[\pi-x, \sigma_{1}(t, \tau)\right] d \tau \\
& +\int_{0}^{t} C_{3}(\tau) \exp \left[-\sigma_{2}(t, \tau)\right] \frac{\partial}{\partial \tau} U_{0}\left[x, \sigma_{1}(t, \tau)\right] d \tau \\
& +\frac{1}{2} \exp \left[-s_{2}(t)\right] \int_{-\pi}^{\pi} F(x-\xi) \frac{\partial}{\partial \xi} U_{0}\left[\xi, s_{1}(t)\right] d \xi .
\end{aligned}
$$

4. Verification of resolution. Instead of verifying directly that the function given by (3) is a solution of problem (A), we shall take up separately the verifications of the solutions $U_{1}, U_{2}$, and $U_{4}$ of the problems $\left(A_{1}\right),\left(A_{2}\right)$, and $\left(A_{4}\right)$, respectively.

Consider problem $\left(\mathrm{A}_{1}\right)$ and its solution $U_{1}(x, t)$ given by $(2)$. By making use of the definitions of extensions, we can write $U_{1}(x, t)$ as follows:

$$
\begin{aligned}
U_{1}(x, t)= & \frac{1}{2} \int_{0}^{t} \exp \left(-\sigma_{2}\right) \\
& \cdot \int_{0}^{\pi}[P(x+\xi, \tau)+P(x-\xi, \tau)] \frac{\partial}{\partial \xi} U_{0}\left(\xi, \sigma_{1}\right) d \xi d \tau .
\end{aligned}
$$

Integration by parts with respect to $\xi$ yields

$$
U_{1}(x, t)=\frac{1}{2} \int_{0}^{t} \exp \left(-\sigma_{2}\right) H(x, t, \tau) d \tau,
$$

where

$$
H(x, t, \tau)=\int_{0}^{\pi} G(x, \xi, \tau) U_{0}\left[\xi, \sigma_{1}(t, \tau)\right] d \xi
$$

and 


$$
G(x, \xi, \tau)=\left[\frac{\partial}{\partial x} P(x-\xi, \tau)-\frac{\partial}{\partial x} P(x+\xi, \tau)\right]
$$

The function $U_{0}(x, t)$, the solution of problem (B), can be found by an inverse sine transformation of $u_{0}(n, t)$, or by the usual methods. ${ }^{5}$ However, this problem is a special case of a more general problem solved by R. V. Churchill ${ }^{6}$ by means of the Laplace transformation. The following properties of $U_{0}(x, t)$ can be derived from his paper, and are given here without the proofs: $U_{0}(x, t)$ is of order $O[\exp (\gamma t)]$ uniformly in $x, 0 \leqq x \leqq \pi$, for all $t \geqq 0$, for some $\gamma>0$; $U_{0}(x, t)$ is continuous in $(x, t)$ when $t \geqq 0,0<x \leqq \pi ; U_{0}, \partial U_{0} / \partial x$, $\partial^{2} U_{0} / \partial x^{2}$, and $\partial U_{0} / \partial t$ are continuous in $(x, t)$ for each $t>0,0 \leqq x \leqq \pi$; $\left|\partial U_{0} / \partial t\right|$ is bounded when $t \geqq 0$ and $x$ is in the interval $0<x_{0} \leqq x \leqq \pi$.

That $H(x, t, \tau), \partial H(x, t, \tau) / \partial t$, and $\partial^{2} H(x, t, \tau) / \partial x^{2}$ are continuous functions of $(x, t, \tau)$ in $R^{\prime}, 0 \leqq x \leqq \pi, 0 \leqq \tau \leqq t$, can be established as follows:

$F \equiv G U_{0}$ is a continuous function of $(x, t, \tau, \xi) \equiv(\rho, \xi)$ in the closed region $R^{\prime \prime}: R^{\prime}, \delta_{1} \leqq \xi \leqq \pi, \delta_{1}>0$. Hence $|\Delta F|<\epsilon_{1}$ for $|\Delta \rho|<\delta^{\prime}$ in $R^{\prime \prime}$. $F$ is uniformly bounded and integrable in the closed region $R^{\prime \prime \prime}: R^{\prime}$, $0 \leqq \xi \leqq \delta_{1}$. Hence $|F|<M$ in $R^{\prime \prime \prime}$. So that

$$
\begin{aligned}
|H(\rho+\Delta \rho)-H(\rho)| & \leqq \int_{0}^{\delta_{1}}|\Delta F| d \xi+\int_{\delta_{1}}^{\pi}|\Delta F| d \xi \\
& \leqq M \delta_{1}+\epsilon_{1}\left(\pi-\delta_{1}\right)<\epsilon
\end{aligned}
$$

for $|\Delta \rho|<\delta<\delta^{\prime}, \rho$ in $R^{\prime}$. This establishes the continuity of $H(x, t, \tau)$. Consider

$$
\frac{\partial}{\partial t} H(x, t, \tau)=\frac{\partial}{\partial t} \int_{0}^{\pi} G(x, \xi, \tau) U_{0}\left[\xi, \sigma_{1}(t, \tau)\right] d \xi .
$$

The derivative of the integral with respect to $t$ yields formally

$$
\begin{aligned}
\frac{\partial}{\partial t} H(x, t, \tau) & =\int_{0}^{\pi} G(x, \xi, \tau) \frac{\partial}{\partial t} U_{0}\left[\xi, \sigma_{1}(t, \tau)\right] d \xi \\
& =C_{1}(t) \int_{0}^{\pi} G(x, \xi, \tau) \frac{\partial^{2}}{\partial \xi^{2}} U_{0}\left(\xi, \sigma_{1}\right) d \xi \\
\frac{\partial}{\partial t} H(x, t, \tau) & =C_{1}(t) \int_{0}^{\pi} \frac{\partial^{2}}{\partial \xi^{2}} G(x, \xi, \tau) U_{0}\left(\xi, \sigma_{1}\right) d \xi .
\end{aligned}
$$

${ }^{5}$ R. V. Churchill, Fourier series and boundary value problems, New York, McGrawHill Book Company, 1941, p. 102.

${ }^{6} \mathrm{R}$. V. Churchill, On the problem of temperatures in a non-homogeneous bar with discontinuous initial temperatures, Amer. J. Math. vol. 61 (1939) pp. 651-664. 
This integral is of the type $H(x, t, \tau)$, therefore $\partial H(x, t, \tau) / \partial t$ is a continuous function of $(x, t, \tau)$ in $R^{\prime}$, provided $\partial H(x, t, t) / \partial t$ is defined by (d). The continuity of $\partial^{2} H(x, t, \tau) / \partial x^{2}$ can be established by the same type of argument.

Consider $U_{1}(x, t)$ as defined by formula (a). Since the integrand is a bounded, integrable function of $\tau$ for all $t \geqq 0$, the limit of $U_{1}(x, t)$ as $t$ approaches zero exists and $U_{1}(x,+0)=0$.

Since $G(x, \xi, \tau)$, in (c), vanishes when $x$ approaches both end points of $(0, \pi)$, it follows that $H(0, t, \tau)=H(\pi, t, \tau)=0$. Hence $U_{1}(+0, t)=0$ and $U_{1}(\pi-0, t)=0$.

To show that $U_{1}(x, t)$ satisfies the differential equation of problem $\left(\mathrm{A}_{1}\right)$, we apply Leibnitz' rule to $U_{1}(x, t)$, in (a), and find that

$$
\begin{aligned}
\frac{\partial}{\partial t} U_{1}(x, t)= & \frac{1}{2} \lim _{\tau \rightarrow t} \exp \left(-\sigma_{2}\right) H(x, t, \tau) \\
& +\frac{1}{2} \int_{0}^{t} \frac{\partial}{\partial t}\left\{\exp \left[-\sigma_{2}(t, \tau)\right] H(x, t, \tau)\right\} d \tau \\
= & \frac{1}{2} \int_{0}^{\pi} G(x, t, t) U_{0}(\xi, 0) d \xi-C_{2}(t) U_{1}(x, t) \\
& +\frac{1}{2} \int_{0}^{t} \exp \left(-\sigma_{2}\right) \frac{\partial}{\partial t} H(x, t, \tau) d \tau .
\end{aligned}
$$

Since $\partial^{2} G / \partial x^{2}=\partial^{2} G / \partial \xi^{2}$, we have from (d) that $\partial H / \partial t=C_{1} \partial^{2} H / \partial x^{2}$. Hence

so that

$$
\begin{aligned}
\frac{\partial}{\partial t} U_{1}(x, t)= & P(x, t)-C_{2}(t) U_{1}(x, t) \\
& +C_{1}(t) \frac{1}{2} \int_{0}^{t} \exp \left(-\sigma_{2}\right) \frac{\partial^{2}}{\partial x^{2}} H(x, t, \tau) d \tau,
\end{aligned}
$$

$$
\begin{aligned}
\frac{\partial}{\partial t} U_{1}(x, t)=P(x, t)-C_{2}(t) U_{1}(x, t)+C_{1}(t) & \frac{\partial^{2}}{\partial x^{2}} U_{1}(x, t), \\
0 & <x<\pi, t>0 .
\end{aligned}
$$

Consider problem $\left(\mathrm{A}_{2}\right)$ and its solution $U_{2}(x, t)$ given by (2). Since $C_{3}(0)=0$, integration by parts of $U_{2}(x, t)$ yields

$$
\begin{aligned}
U_{2}(x, t) & =C_{3}(t)((\pi-x) / \pi) \\
- & \int_{0}^{t} \exp \left[-\sigma_{2}(t, \tau)\right] U_{0}\left[x, \sigma_{1}(t, \tau)\right]\left\{C_{3}^{\prime}(\tau)+C_{2}(\tau) C_{3}(\tau)\right\} d \tau,
\end{aligned}
$$


so that $U_{2}(x,+0)=0,0<x<\pi$.

Now $\left|U_{0}\right|<\epsilon^{\prime}$ when $0<x<\delta^{\prime}, 0 \leqq \tau \leqq t-\epsilon^{\prime \prime} ;\left|U_{0}\right|<\left|(\pi-x) / \pi+\epsilon^{\prime \prime \prime}\right|$ when $0<x<\delta^{\prime \prime}, t-\epsilon^{\prime \prime} \leqq \tau \leqq t$; so that if $E \equiv \exp \left(-\sigma_{2}\right)\left[C_{3}^{\prime}+C_{2} C_{3}\right]$, $|\max E|=M$, then

$$
\begin{aligned}
&\left|U_{2}(x, t)-C_{3}(t)\right| \leqq\left|C_{3} / \pi\right| x+\left|\int_{0}^{t} E U_{0} d \tau\right| \\
& \leqq\left|C_{3}\right| x+\left|\int_{0}^{t-\epsilon^{\prime \prime}} M \epsilon^{\prime} d \tau\right| \\
&+\left|\int_{t-\epsilon^{\prime \prime}}^{t} M\right|(\pi-x) / \pi+\epsilon^{\prime \prime \prime}|d \tau| \\
& \leqq\left|C_{3}\right| x+M\left(t-\epsilon^{\prime \prime}\right) \epsilon^{\prime}+M\left|1+\epsilon^{\prime \prime \prime}\right| \epsilon^{\prime \prime}<\epsilon
\end{aligned}
$$

for $0<x<\delta, \delta<\delta^{\prime}, \delta^{\prime \prime}, 0<t \leqq T$. Hence $U_{2}(+0, t)=C_{3}(t), t>0$. The same type of argument gives $U_{2}(\pi-0, t)=0$, since $U_{0}(\pi-0, t)=0$, $t>0$.

In (e), for $t>0,0<x<\pi$, first we take the derivative of $U_{2}(x, t)$ with respect to $t$, and then we take the second derivative of $U_{2}(x, t)$ with respect to $x$. We find that

$$
\begin{gathered}
\partial U_{2}(x, t) / \partial t=C_{3}^{\prime}(t)((\pi-x) / \pi)-((\pi-x) / \pi)\left[C_{3}^{\prime}(t)+C_{2}(t) C_{3}(t)\right] \\
+C_{2}(t) \int_{0}^{t} \exp \left(-\sigma_{2}\right) U_{0}\left(x, \sigma_{1}\right)\left[C_{3}^{\prime}(\tau)+C_{2}(\tau) C_{3}(\tau)\right] d \tau \\
-C_{1}(t) \int_{0}^{t} \exp \left(-\sigma_{2}\right)\left(\partial U_{0}\left(x, \sigma_{1}\right) / \partial \sigma_{1}\right)\left[C_{3}^{\prime}(\tau)+C_{2}(\tau) C_{3}(\tau)\right] d \tau,
\end{gathered}
$$

and

$\partial^{2} U_{2}(x, t) / \partial x^{2}$

$$
=-\int_{0}^{t} \exp \left(-\sigma_{2}\right)\left(\partial^{2} U_{0}\left(x, \sigma_{1}\right) / \partial x^{2}\right)\left[C_{3}^{\prime}(\tau)+C_{2}(\tau) C_{3}(\tau)\right] d \tau .
$$

We form the partial differential expression $L\left(U_{2}\right)$ :

$$
\begin{aligned}
L\left(U_{2}\right)= & \partial U_{2} / \partial t-C_{1}(t) \partial^{2} U_{2} / \partial x^{2}+C_{2}(t) U_{2} \\
& =C_{1}(t) \int_{0}^{t} \exp \left(-\sigma_{2}\right)\left[\partial^{2} U_{0}\left(x, \sigma_{1}\right) / \partial x^{2}\right. \\
& \left.\quad-\partial U_{0}\left(x, \sigma_{1}\right) / \partial \sigma_{1}\right]\left[C_{3}^{\prime}+C_{2} C_{3}\right] d \tau .
\end{aligned}
$$

Hence $L\left(U_{2}\right)=0$, since $\partial^{2} U_{0}\left(x, \sigma_{1}\right) / \partial x^{2}-\partial U_{0}\left(x, \sigma_{1}\right) / \partial \sigma_{1}=0,0<x<\pi$, $t>0$. 
The verification of $U_{4}(x, t)$ can be patterned after that used for $U_{1}(x, t)$ and, therefore, will not be discussed here.

This concludes the verification of the resolution of problem (A). The restrictions placed upon the functions $F(x)$ and $P(x, t)$ are unnecessarily severe. They were made in order to be able to condense the forms of the solutions. The same applies to the condition that $C_{3}(0)=C_{4}(0)=0$. The verification of $U_{3}$ and $U_{4}$ can be made without them.

5. Temperature distribution in a cube. The method of the previous problem can be extended to a general heat flow problem in three dimensions; the same problem (B) is used in the resolution. The verification of the resolution of this problem is similar to that of the first problem and is omitted here.

The interior of a cube $\mathrm{c}^{7}$ of edge length $\pi$ is filled with a homogeneous, isotropic medium. The temperatures on the faces of the cube are maintained at prescribed values, depending upon the space coordinates of the point and upon the time. There is a continuous source of heat in the medium whose thermal conductivity may be a function of the time. Further, at each point of the medium there is a sink of heat which may be a function of the time and which is proportional to the temperature at that point. The initial temperature distribution is given by a prescribed function. We take a corner of the cube as origin and the three mutually perpendicular edges as coordinate axes to locate a point $P:\left(x_{1}, x_{2}, x_{3}\right)$ of the cube.

Let us consider the distribution of temperatures $U(P, t)$ in the interior of such a cube. $U(P, t)$ satisfies the conditions:

$$
\begin{array}{rr}
\left.\frac{\partial}{\partial t} U(P, t)\right\rceil-\sum_{j=1}^{3} C_{j}(t) \frac{\partial^{2}}{\partial x_{1}^{2}} U(P, t)-C_{4}(t) U(P, t)=F(P, t), & 0<x_{j}<\pi, t>0, \\
U\left(0, x_{2,3}, t\right)=G_{1}\left(x_{2,3}, t\right), U\left(\pi, x_{2,3}, t\right)=G_{2}\left(x_{2,3}, t\right), 0 \leqq x_{2,3} \leqq \pi, t>0, \\
U\left(0, x_{1,3}, t\right)=G_{3}\left(x_{1,3}, t\right), U\left(\pi, x_{1,3}, t\right)=G_{4}\left(x_{1,3}, t\right), 0 \leqq x_{1,3} \leqq \pi, t>0, \\
U\left(0, x_{1,2}, t\right)=G_{5}\left(x_{1,2}, t\right), U\left(\pi, x_{1,2}, t\right)=G_{6}\left(x_{1,2}, t\right), 0 \leqq x_{1,2} \leqq \pi, t>0, \\
U(P, 0)=H(P), & 0<x_{j}<\pi(j=1,2,3) .
\end{array}
$$

Problem (B), which is a problem in heat conduction in one dimension, is sufficient to make a formal resolution of this very general problem of heat conduction in three dimensions.

${ }^{7}$ Compare with problem discussed by Carslaw: H. S. Carslaw, Introduction to the mathematical theory of the conduction of heat in solids, London, Macmillan and Company, 1921, p. 108. 
If we use the same system of abbreviations as that used before, the solution $U(P, t)$ can be expressed as follows:

$$
\begin{aligned}
U(P, t)=\frac{1}{8} \int_{0}^{t} \exp \left(-\sigma_{4}\right) \iiint_{-\pi}^{\pi} F\left(P_{j}, \tau\right) \prod_{j=1}^{3} \frac{\partial}{\partial \xi_{j}} U_{0}\left(\xi_{j}, \sigma_{j}\right) d \xi_{j} d \tau \\
+\frac{1}{8} \exp \left(-s_{4}\right) \iiint_{-\pi}^{\pi} H\left(P_{j}\right) \prod_{j=1}^{3} \frac{\partial}{\partial \xi_{j}} U_{0}\left(\xi_{j}, s_{j}\right) d \xi_{j} \\
+\frac{1}{4} \sum_{j=1}^{3} \int_{0}^{t} \exp \left(-\sigma_{4}\right) \frac{\partial}{\partial \tau} U_{0}\left(x_{j}, \sigma_{j}\right) \\
+\iint_{-\pi}^{\pi} G_{2 j-1}\left(Q_{j}, \tau\right) \prod_{k \neq j=1}^{3} \frac{\partial}{\partial \xi_{k}} U_{0}\left(\xi_{k}, \sigma_{k}\right) d \xi_{k} d \tau \\
+\frac{1}{4} \sum_{j=1}^{3} \int_{0}^{t} \exp \left(-\sigma_{4}\right) \frac{\partial}{\partial \tau} U_{0}\left(\pi-x_{j}, \sigma_{j}\right) \\
\cdot \iint_{-\pi}^{\pi} G_{2_{j}}\left(Q_{j}, \tau\right) \prod_{k \neq \neq}^{8} \frac{\partial}{\partial \xi_{k}} U_{0}\left(\xi_{k}, \sigma_{k}\right) d \xi_{k} d \tau,
\end{aligned}
$$

where $\left(P_{j}\right) \equiv\left(x_{1}-\xi_{1}, x_{2}-\xi_{2}, x_{3}-\xi_{3}\right)$ and $Q_{j}$ is of the form $\left(x_{j_{1}}-\xi_{j_{1}}\right.$, $\left.x_{j_{2}}-\xi_{j_{2}}\right) ; j_{1}, j_{2} \neq j=1,2,3$.

Rensselaer Polytechnic Institute 